\title{
Finite Element Vibration Analysis of Damped Composite Sandwich Beams
}

\author{
Samir Assaf \\ Ecole Supérieure des Techniques Aéronautiques et de Construction Automobile (ESTACA), Lab. Acoustique \\ et Vibration, 34, rue Victor Hugo, 92532 Levallois Perret Cedex, France
}

(Received 1 October 2010; provisionally accepted 26 July 2011; accepted 13 October 2011)

In this study, a finite element model for the vibration analysis of cross-ply laminated sandwich beams is presented. This formulation is an extension of our previous work on predicting the acoustic and vibration responses of sandwich beams and plates with homogeneous elastic faces and a viscoelastic core. The formulation is based on a layerwise linear axial displacement through the beam thickness. The formulation assumes the classical lamination theory for the faces and Timoshenko theory for the core. The governing equations of motion are obtained using Hamilton's principle. A finite element method and a beam element are further developed to predict the natural frequencies and modal loss factors. In order to validate the proposed model, several free vibration analyses of composite sandwich beams with different boundary conditions, length-to-thickness ratios and face laminations are presented. The results are then compared with solutions available in the literature and with those obtained by the PATRAN/NASTRAN finite element software package. These results show the validity of the present formulation. Finally, the effects of ply-stacking sequence, core-to-face stiffness ratio and core-to-face thickness ratio upon the natural frequencies and modal damping are investigated.

\section{INTRODUCTION}

Laminated composite sandwich structures are widely used in the design of structures in the aero-nautics and aerospace industries due to their high specific stiffness and high specific strength. In order to reduce vibration and noise, the damping in the core and in the composite materials is an important parameter in the design of such structures. A common way to enhance the damping cha-racteristics of structures is the use of constrained viscoelastic layer treatment. ${ }^{1}$ In these constrained configurations, the core material is subjected primarily to shear loading caused by relative in-plane displacements of the constraining layer and the base layer. Damping is induced by the high transverse shear deformations of the viscoelastic layer, which are accompanied by high energy dissipations.

Sandwich structures have been studied by many authors. Various displacement-based theories have been proposed according to the type of sandwich materials and geometric configurations. These theories differ mainly in the inclusion of the effects of the shear strain in their kinematic formulations. The classical laminate theory (CLT) is based on Kirchhoff thin plate assumptions, and it neglects the transverse shear strains. The inclusion of transverse shear strains leads to the first order shear deformation theory (FSDT). This model is known as the Mindlin plate theory, and it assumes a constant transverse shear strain in the thickness direction. It consists of a first-order Taylor-type expansion of unknown displacements. FSDT cannot take into account the variation in the shear strain through the thickness predicted by the 3-D elasticity theory. This leads to the development of higher-order displacement models-based on a Taylor-type series expansion-known as higher-order shear deformation theories (HSDT). Due to the different mechanical-physical properties in the thickness direction, higher-order theories do not fulfill the interlaminar continuity of transverse stresses at each layer interface. Layerwise or Zig-Zag theories were introduced to satisfy this inter- laminar continuity of transverse shear stresses and to describe accurately their variation through the thickness.

Reviews and assessments of various kinematics and theories used for the modeling of sandwich composites can be found in references 2-5. Carrera and Ciuffreda used a unified formulation to compare about forty theories for multilayered, composite, and sandwich plates that are loaded by transverse pressure with various in-plane distributions (harmonic, constant, triangular, and tent-like). ${ }^{3}$ Layerwise and equivalent single-layer models related to linear and higher-order expansions in the plate thickness direction were considered. W. Zhen and C. Wanji assessed several displacement-based theories by analyzing the free vibration and the buckling behavior of laminated beams with arbitrary layouts as well as soft-core sandwich beams. ${ }^{4}$ FSDT, ${ }^{6}$ HSDT, ${ }^{7-9}$ as well as a Zig-Zag theory ${ }^{10}$ were considered. $\mathrm{H}$. Hu et al. ${ }^{5}$ proposed a unified kinematic formulation to analyze and assess CLT, HSDT, ${ }^{7,11}$ and Zig-Zag theories. ${ }^{12,13}$ Static and dynamic problems were considered and analyzed for various sandwich structure configurations.

In previous work, a displacement-based finite element formulation was proposed by the author to predict the acoustic and vibration response of sandwich plates with homogeneous elastic faces and a viscoelastic core. ${ }^{14}$ This plate formulation is based on Kirchhoff's theory for the elastic faces and on Mindlin's theory for the core. The nodal degrees of freedom associated with the displacement field are the mean and relative in-plane displacements of the faces and the transverse deflection of the plate. In addition to its ease of formulation and coding, this model requires reasonable computational time and cost, and it accurately predicts the global vibration and acoustic response of arbitrarily shaped three-layer plates under various boundary conditions (i.e., mode shapes, natural frequencies and associated modal loss factors, mean quadratic velocity, radiated sound power, diffuse sound field transmission loss). ${ }^{14,15}$ 